\title{
Enabling Users to Create and Document 3D Content for Heritage
}

\author{
Karina Rodriguez-Echavarria, David Morris, David Arnold \\ Faculty of Management and Information Sciences, University of Brighton, UK \\ Email:K.Rodriguez@brighton.ac.uk
}

\begin{abstract}
This paper presents research on developing processes for content creation of $3 \mathrm{D}$ assets of public monuments and sculptures in towns and cities in the Sussex area of the United Kingdom. This type of heritage is usually located in neighbourhoods among the public, who live and interact with them on a day to day basis. The paper recognises the challenges involved in requiring non-experts to use software and hardware for 3D graphics, which are not yet of common use. Hence, the research has been focused from early stages to gather feedback in order to lower the threshold of 3D technologies. Specifically, this paper addresses the development and use of tools to allow users to create 3D content and integrate it with semantic information. The information is disseminated in a web based format. As a result, the public sculptures and monuments database supports scholar and public dissemination by presenting a variety of integrated documentation on the project website: http://www.publicsculpturesofsussex.co.uk
\end{abstract}

Keywords-User generated content; Computer graphics software; Knowledge acquisition; User interfaces

\section{INTRODUCTION}

For the past few years, the Public Monuments and Sculpture Association (PMSA) in the United Kingdom has endeavoured to record all public monuments and sculptures under their remit. For this, the country was divided into Regional Archive Centres (RACs), mostly run from academic institutions; each responsible for the survey and digitised record of its local area. The two-year, Heritage Lottery Funded, National Recording Project for Sussex undertook to survey from all periods, conventional statues, monumental works, selected war memorials, architectural sculpture, contemporary sculpture, memorial clocks, towers and fountains. Objects that were 'lost' were also included.

The main aims of the recording process have been twofold; to provide a dynamic, accurate digital "catalogue" of existing and new pieces that can be used by scholars and the general public as an information source, and, to foster a growing interest amongst communities in the sculptural heritage of their local area. The initial "catalogue" contains information mainly in text format and photographs recorded by a qualified researcher.

This paper describes the research conducted as a continuation of this project. As a recognition that the public has a great interest in learning about objects that they interact with on a day to day basis, the aim of this work is to empower users to become active contributors of heritagerelated content. Hence, the paper acknowledges that end- users' contributions will be critical for acquiring and documenting 3D resources, just as it is now for images and videos in the web. Nevertheless, it is recognised that there are challenges involved in requiring non-experts to use software and hardware for 3D graphics which are not yet of common use. To address this, the research has been focused from early stages to gather feedback in order to lower the threshold of 3D technologies. The main challenges involve designing processes which can be followed by users with a minimum amount of training. The final aim is to enable users to capture and document $3 \mathrm{D}$ representations of the objects in the PMSA catalogue and their metadata.

Section II introduces 3D technologies for museums; while Section III describes the data collected during the survey and its re-purposing to serve as metadata. Sections IV and $\mathrm{V}$ describe the planning and testing of the 3D digitisation exercises, followed by section VI with conclusions and further work.

\section{3D TECHNOLOGIES FOR HERITAGE}

The use of 3D technologies for heritage is an interdisciplinary area with fast-paced developments in the last 10-15 years. 3D technologies are increasingly being used to capture, document and present artefacts and sites more accurately than by just using images and text. Visitors to heritage institutions also benefit from 3D reconstructions to support their interpretation of the historical material in display. This is particularly important when heritage artefacts are fragile and not easily available for people to see, touch and understand its relevance. Hence, most research in the area focuses in making tools for data acquisition, processing, documentation, visualisation and augmentation more accessible and reliable for the sector ([1], [2]).

Further research in the area, such as [3], addresses the need of enabling and encouraging end-users to act as contributors of content for this sector. This requires i) making tools (software and hardware) easy to use and ii) processes which can be scaled up for larger groups of relatively untrained people. This will be very beneficial for collections, such as the PMSA catalogue; as only a large coverage of acquisition and documentation will enable $3 \mathrm{D}$ content to be produced. The following sections will describe initial efforts to achieve this. 


\section{RECORDING INFORMATION OF SCULPTURES AND MONUMENTS}

The initial data collection took place through a detailed surveying exercise based on a pro-forma document that was established by the PMSA. This document was used during on-site visits to each of the objects. A detailed description of the object was recorded as well as the objects' general history, including that of inauguration events, unveiling ceremonies. A concise note of the condition of the object was made and this is particularly useful for conservators, especially in the case of listed objects. This listing system is used in the UK for protection by marking an object's special architectural and historic interest.

The location of the objects was precisely recorded by latitude and longitude to offer a range of geographical information system (GIS) functionalities within the website. A rich image archive was also recorded involving photographs of signatures, architectural details and specific damage. No judgements were made with regard to the aesthetic worth of an object, indeed, many objects included could be described as vernacular sculpture or functional street furniture. Other objects were recorded that may on examination fall short of being categorised as monuments or sculpture but which may have a particularly interesting historical significance or inscription for instance.

All objects were visited by the survey team and there was no reliance on secondary sources for the details of the physical survey. This was to avoid repeating abundant errors with regard to inscriptions, for example, that can be found in the many scholarly and other texts on the subject. Books, the Internet and archival sources such as local newspapers, were used to gather details on the historical context of the object and, where possible, direct contact was made with the sculptors and artists involved in its creation. With an emphasis on data quality monitoring, in addition to the Project Research Officer, Anthony McIntosh, only one trained volunteer, Gina Lelliott, was recruited and trained to carry out surveys.

The information collected during the survey was stored within a relational database implemented in a MySQL database. Currently the database holds around 450 objects. The results from this stage included a database accessible via the website: http://www.publicsculpturesofsussex.co.uk (see figure 1). This was developed using the content management system Drupal, which handles typical functions such as theming and authentication.

\section{A. Generating semantic metadata using CIDOC-CRM on- tology}

Semantic web technologies were used to generate a metadata repository using the relational database produced. For this, the Resource Description Framework (RDF) [4] language was used as a basis to describe the elements in

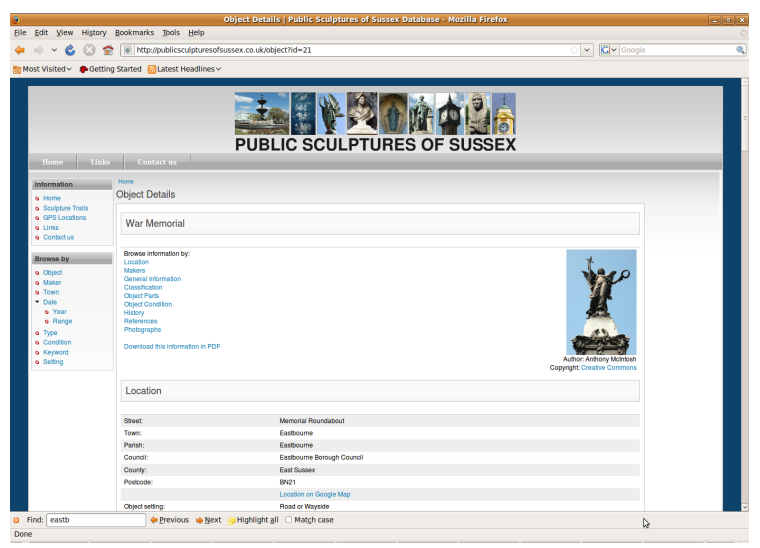

Figure 1. Sussex public sculptures and monuments website

the database using the CIDOC-CRM ontology [5]. CIDOC$\mathrm{CRM}$ is a formal ontology which facilitates the integration and interchange of heterogeneous cultural heritage information. One of the main characteristic of this ontology is that it analyses the past dividing it into discrete events involving persistent items, both material and immaterial.

Furthermore, the ontology is being extended to describe the provenance for both physical and digital objects. In this context, provenance describes the events that occur during a digital object's life cycle including the process of digitising and documenting the $3 \mathrm{D}$ content. This information is important to record the provenance of the $3 \mathrm{D}$ content [6]. Hence, it is possible to record important information such as: who made the digitisation?; which were the settings of the hardware?; who made the post-processing of the 3D content?, who interpret the documentation? A further challenge will be to enable volunteers who which to remain anonymous while still recording provenance information which could help professionals and public alike to understand where the information comes from.

The D2R Server tool [7] was used for publishing the content of the MySQL database in RDF. A mapping file in the Notation3 language was developed to allow the RDF data to be browsed and searched within the CIDOC-CRM context. Notation3 is equivalent to RDF in its XML syntax, but is more compact and readable.

Figure 2 illustrates a sample of the CIDOC-CRM based data model for structuring the information on the metadata repository. This data model maps the most important concepts regarding the sculptures and monuments (e.g. E84.Information_Carrier, E55.Event, E3.Condition_state, E39.Actor, E26.Physical_feature).

The Notation3 mapping file provided a translation for each of the relevant concepts in the database to CIDOCCRM complaint concepts. The advantage of this approach is that only one database needs to be maintained for the whole framework. In addition, D2R provides a mechanism to 


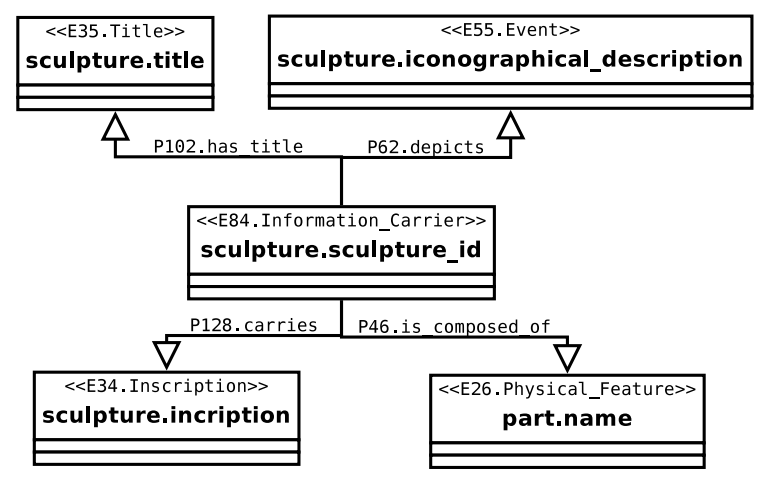

Figure 2. Sample of the CIDOC-CRM mapping data model for storing metadata about sculptures and monuments.

query the metadata repository over the Web via the SPARQL protocol and as linked data. The SPARQL protocol was developed by the W3C RDF Data Access Working Group (DAWG) and is a query language and protocol for RDF. In this protocol data is exposed and shared via dereferenceable URIs on the Web. As a result, the information in the PMSA database was ready to be used as metadata.

\section{Designing PRocesses to ENABLE USERS TO CONTRIBUTE TO THE REPOSITORY}

The public in UK towns and cities experience sculptures and monuments as objects they interact with on a day to day basis. Nevertheless, their participation during the documentation phase was almost non-existent. Up to this stage, it was regarded as important that all the information recorded was accurate with existing reference and bibliographical sources. Hence, only the researchers within the project were involved. However, the fact that most objects in the PMSA remit are three dimensional made this collection suitable for tapping on the potential of the public generating 3D content to enrich the documentation. Hence, it was necessary to research ways on which the public could "easily" contribute to the repository by volunteering to acquire and document 3D content.

Although the acquisition of 3D models of the objects was the main focus, the documentation of connections between the $3 \mathrm{D}$ model with information available in the metadata repository was considered also critical. The reason is that $3 \mathrm{D}$ content without the context of what is being represented is not very useful for users. Indeed, it was regarded important to demonstrate the value of $3 \mathrm{D}$ content for contextualising other information in the database. This will benefit i) general users, as not everybody has the same expertise for reading lengthy descriptions on the physical parts, history and iconographic meaning of each of the parts of the sculpture; and ii) scholars, who will be able to explore new methods to investigate and discover objects' relevance and their context. For example, finding connections, not explored before, within the documentation of the sculptures.

The main challenge to address was to identify an efficient processes which could be followed by users with a minimum amount of training and little or no intervention from the curator on a large scale basis. In order to achieve this, a quality cycle methodology with iterative steps was selected:

1) Planning: this includes the initial stages of defining a strategy for the testing, selecting the objects for digitisation, recruiting volunteers, selecting the techniques and providing the preparatory training.

2) Deploying: this step involves defining and testing the process users need to follow to create content, including acquiring, processing, documenting, managing and presenting the $3 \mathrm{D}$ content.

3) Assessing: this includes giving feedback on tools, process and effectiveness of the process. This step is critical if the tools are to improve to a stage in which they can be easily used by the general public.

It should be highlighted that the tools and the process considered are experimental; hence they will require constant feedback and improvement. This is why the iterative nature of the cycle means that the research was first conducted with an initial set of objects and a limited number of volunteers with a higher level of graphic skills. These restrictions will iteratively disappear as the process and tools get developed further.

\section{TESTING PROCESSES FOR ACQUIRING AND DOCUMENTING 3D CONTENT}

The strategy for the initial testing cycle was to select a small range of objects and tools; with which the team could study the feasibility of the process and the amount of resources required for testing the process on a bigger scale. Three objects were selected within the city of Brighton and the town of Petworth. These objects are illustrated in figure 3. They include a mixture of scales, shapes, complexity and settings on which they are placed.

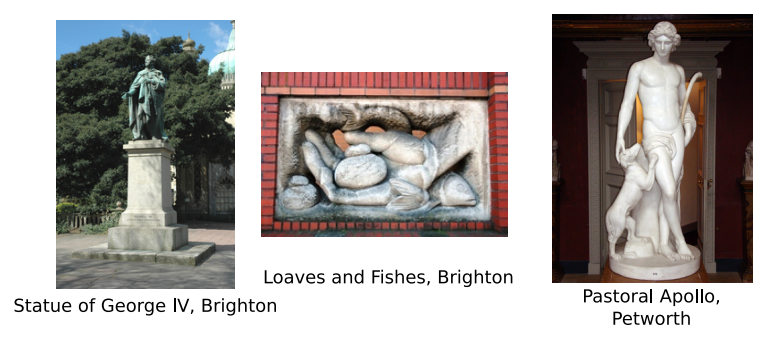

Figure 3. Objects selected for initial testing in Brighton and Petworth.

A team of three researchers were involved and two techniques for acquiring 3D information were considered:

- Laser scanning: This process requires a laser scanner and expertise in handling the hardware and processing 
the 3D information. As this technique is more time consuming, expensive and requires of specialist knowledge; it is expected that it could only be deployed by technical volunteers in controlled environments.

- Photogrammetry techniques: This process requires a digital camera and low to medium expertise in taking the photographs and processing the $3 \mathrm{D}$ information. It is expected that this method could be deployed on a larger scale basis.

The Perceptron Scanworks V4 ${ }^{\mathrm{TM}}$ sensor on a Cimcore infinite arm ${ }^{\mathrm{TM}}$ was used for scanning the "Pastoral Apollo" object at the Petworth Gallery. The scanning was done while the gallery was closed to the public during 2 days. The information acquired was later processed using the Scanworks software to produce a 3D mesh.

In the case of the "Statue of George IV" and "Loaves and Fishes" sculptures in the city of Brighton the photogrammetry technique was used. For this, a digital camera, the Arc3D service ([8], [9]) and MeshLab [10] were used for their acquisition.

Photogrammetry is a much simpler process to enable nonexperts to acquire 3D information of objects. It usually involves a user photographing an object while walking with the camera in an arc around the object. The minimum amount of required photographs is two; although the more information is available the better the results. The photographs are then uploaded to a server where a $3 \mathrm{D}$ reconstruction of the scene is produced.

The MeshLab software can then be used by the user to clean and produce a simpler version of the $3 \mathrm{D}$ model of the sculpture. It is envisaged that the 3D model will be used for different purposes; hence, it is important to keep several levels of resolution (number of triangles). After studying the most efficient ways of simplifying the mesh, the poisson surface reconstruction approach was selected. This approach builds a simpler mesh resulting from Arc3D containing around 50,000 vertices. The 3D model is then saved as an .obj file. Figure 4 illustrates the mesh acquired for the "Loaves and Fishes" sculpture after it has been post processed using MeshLab.

After acquiring the 3D model, it is necessary to enable users to document with metadata the 3D model. The integration of current semantic web technologies with 3D graphics is still a challenging area. Previous approaches include adding semantic annotations to areas of the 3D model [12], for instance to describe what the geometry represents. For example, [13] proposed an approach for associating semantic information to 3D worlds using the X3D language.

In addition, [14] describes an authoring tool for creating semantically enriched 3D objects using the CIDOC-CRM ontology. This approach generates a Collada based scene description which includes the 3D model along with semantic annotations which are linked to mark-up areas in the model. The ideas behind this research were used in order to tag

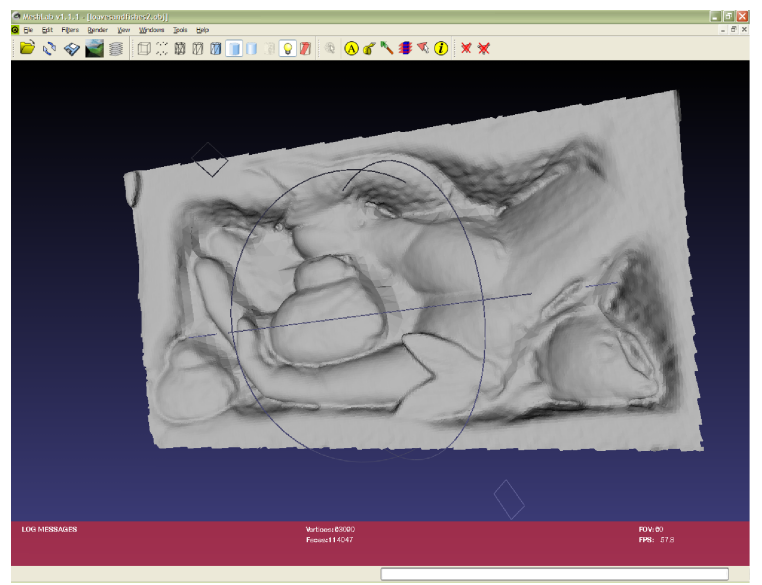

Figure 4. "Loaves and Fishes" sculpture 3D model after post-processing stage.

and store the 3D data; while improving the mechanisms to achieve this. As such, an open source annotation tool called Tagg3D was developed for tagging semantic links to the 3D meshes acquired by the users. The tool was developed using QT4 and the scenegraph API OpenSG2 [15], which is a portable scenegraph system to create real time graphics programs.

Figure 5 illustrates a user case describing the series of steps a user will need to perform to interactively attach URIs to parts of the 3D model. This user case includes different components including Tagg3D and a metadata interface using the Soprano library. This library provides a $\mathrm{C}++/ \mathrm{Qt} 4$ framework for querying and manipulating RDF data. In addition, the metadata databases are accessed using the SPARQL interface. The steps to tag a 3D geometry are:

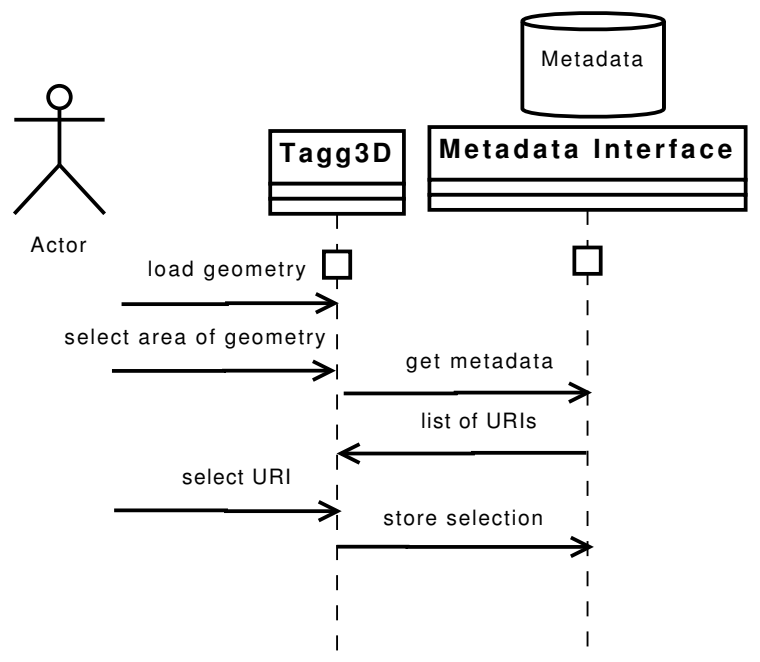

Figure 5. User case for tagging metadata to 3D geometry. 
1) The user loads the geometry.

2) The user selects all or an area of the geometry on which he/she is interested in documenting with metadata. The selection uses a painting mechanism (see figure 6).

3) Tagg3D requests metadata from the repository using a Soprano based metadata interface.

4) The Soprano based interface return a list of URIs of CIDOC-CRM concepts in the metadata repository.

5) The user select a URI which is relevant to the geometry selected. In case that the user has any doubts of what the 3D object (or any of its parts) is representing, communication tools (e.g. chat, audio, video) will enable the communication with experts in the subject area.

6) Tagg3D stores the area of the geometry that the user has selected along with provenance data.

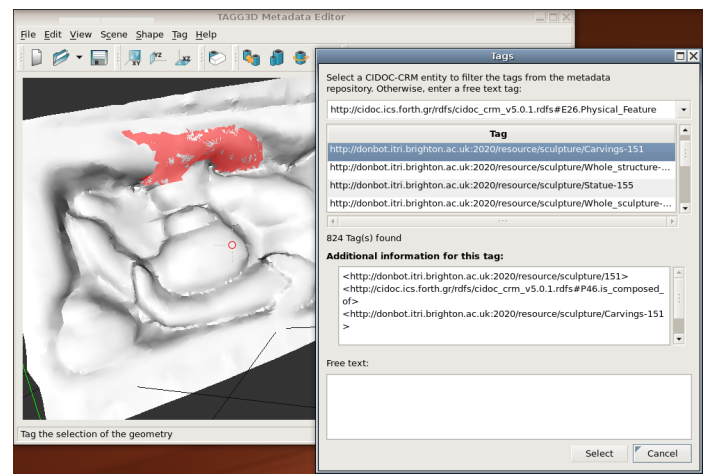

Figure 6. User tagging an area of the 3D geometry using the Tagg $3 \mathrm{~d}$ tool.

As illustrated in figure 6, Tagg3D produces a list of tags which the user can select from (all of them valid URIs in the metadata repository). Additionally, it is possible to use free text as tags. The latter is not recommended as it will not be possible to enquire more information regarding the tag in the repositories.

Internally, the tags are stored within the scenegraph of the geometry as shown in figure 7 . From a top-down view, the root of the scene holds all 3D geometries within the scene. Each geometry is stored in the tree starting with a transformation that contains the transformation matrix. This node contains an attachment with the unique ID for the geometry. Furthermore, the 3D geometry for the sculpture is stored as a child of the transformation node, along with any other "area selections". Those nodes which represent "area selections" are only rendered when requested by the application; hence do not cause any impact to the performance of the system. Finally, the URIs are stored as XML annotation attachments to nodes in the scene. Hence, the URI can be attached to the whole geometry or an "area selection". Once the user has finished tagging the 3D geometry, it is saved as a Collada file and stored in a repository.

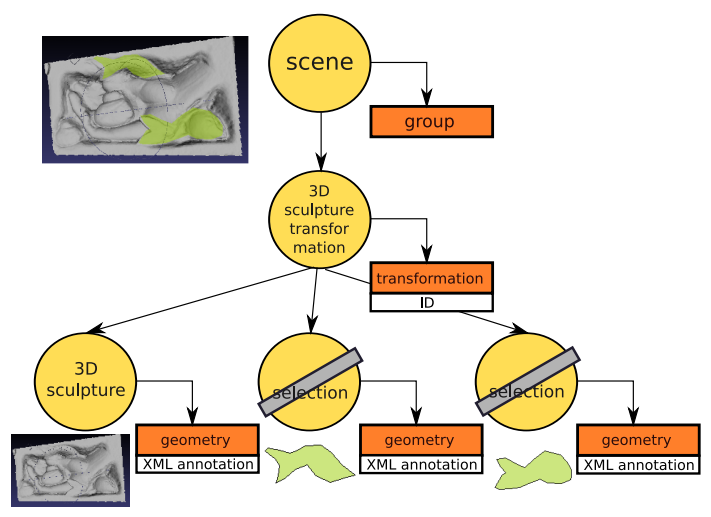

Figure 7. Scenegraph representation of tags within the 3D model.

The work of the user is finished at this stage and the 3D content is ready to be re-used in mashups, visualizations, and other means of presentation. At the moment there is a web plugin implementation to visualise the $3 \mathrm{D}$ representation along with its semantic links in a web broswer [16], as illustrated in figure 8 . Despite the visualisation method, the metadata in the repositories is always queried using the D2R server. This interface enables any application/website to search and query the database using the SPARQL query language over the SPARQL protocol. Within Tagg3D and the Sussex public sculptures and monuments website, the metadata is retrieved by making queries using the URIs. In addition, queries could be done to other repositories with a SPARQL endpoint, such as dbpedia, to access additional information.

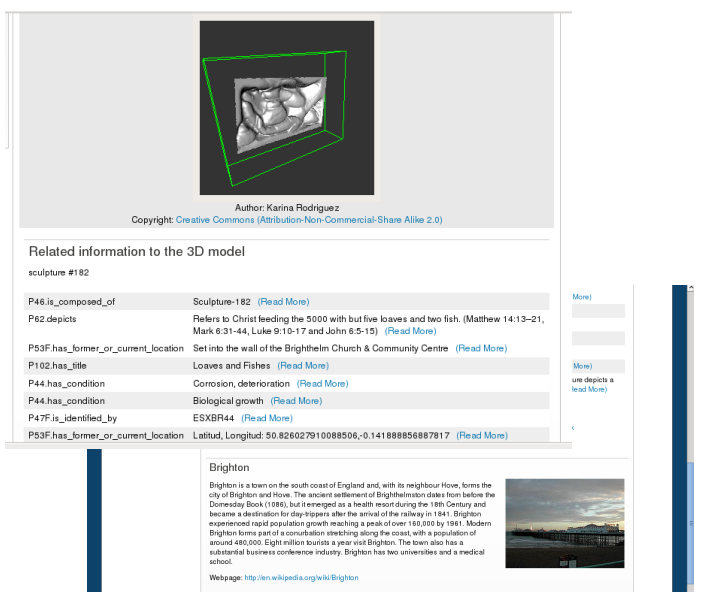

Figure 8. Presenting the 3D content in a webpage

\section{CONCLUSION AND FURTHER WORK}

The initial testing of the process highlighted the potential of the photogrammetry technique for a larger acquisition 
exercise. Nevertheless, the testing also highlighted several challenges with this technique:

- Photogrammetry works better for relieves, than for other types of sculptures, such as statues as these contain a higher level of self occlusion and a higher level of detail.

- Users require minimum training for taking high quality photographs as well as learning to use the Arc3D and Meshlab software. Although, the software is not difficult to use; Meshlab do require a basic understanding of 3D graphics to interact with the 3D model. As such, the photogrammetry and its interface with Meshlab could be improved to achieve a 3D mesh with good quality with a smaller number of photographs so there is less room for mistake.

- A larger number of users as well as data will be more complex to manage. Hence, users will require to have distributed access to a repository and the tools; as well as technical and curatorial expertise in case this is needed.

- Although users have experience with image tagging; 3D mesh tagging requires new navigational and selection skills. This has highlighted the need to automate the process of "selection" of relevant areas in a 3D model (e.g. areas of a body, parts which are in relief).

As a result of the initial testing, feedback on the tools has been compiled and appropriate training is now being developed for the planning of the new set of tests. This is planned to take place in a few months with a larger amount of users and more diverse objects.

Finally, the paper has presented ongoing research to identify and test a process which could be deployed on a large scale basis to acquire and document 3D models of public monuments and sculptures in the UK. Further work will progress by planning the next set of testing, which will take into account improvements to training, deployment processes and the software. It is expected that a larger amount of volunteers will be involved in these testing in order to feedback further exercises until all $3 \mathrm{D}$ content in the database is completed.

\section{ACKNOWLEDGEMENT}

This work has been conducted as part of the 3DCOFORM Integrating Project (FP7-ICT-231809). Thanks to the project team of the National Recording Project for Sussex funded by the Heritage Lottery in collaboration with the Public Monuments and Sculpture Association: Anthony Mcintosh, Peter Seddon and Jill Seddon.

\section{REFERENCES}

[1] D. Arnold and F. Niccolucci and A. Chalmers, VAST 2007: International Symposium on Virtual Reality, Archaeology and Intelligent Cultural Heritage ISBN-978-3-905674-01-9, Brighton, UK
[2] M. Ashley and S. Hermon and A. Proenca and K. RodriguezEchavarria, VAST 2008: International Symposium on Virtual Reality, Archaeology and Intelligent Cultural Heritage ISBN 978-3-905674-14-9, Braga, Portugal

[3] L. Wieneke and J. Nutzel and D. Arnold Life 1.5: Creating A Task Based Reward Structure In Second Life To Encourage And Direct User Created Content Proceedings of ICHIM 2007, October 24-26, 2007, Toronto, Canada

[4] W3C, Resource Description Framework http://www.w3.org/RDF/, visited July 2009.

[5] M. Doerr and D. Iorizzo The dream of a global knowledge network A new approach Journal of Computing and Cultural Heritage, Issue 1, pp.1-23

[6] M. Theodoridou and Y. Tzitzikas and M. Doerr and Y. Marketakis and V. Melessanakis Modeling and Querying Provenance using CIDOC CRM http://www.casparpreserves.eu/Members/metaware/Papers, December 2008

[7] C. Bizer and R. Cyganiak D2R Server http://www4.wiwiss.fuberlin.de, visited July 2009.

[8] M. Vergauwen and L. Van Gool Web-based 3d reconstruction service Machine Vision and Applications. 17, 6, pp.411426, 2006

[9] KU Leuven ESAT-PSI Web3D webpage http://www.arc3d.be/, visited July 2009.

[10] Visual Computing Lab of ISTI-CNR, Meshlab webpage http://meshlab.sourceforge.net/, visited July 2009.

[11] M. Kazhdan and M. Bolitho and H. Hoppe Poisson surface reconstruction. In SGP 06: Proceedings of the fourth $\mathrm{Eu}-$ rographics symposium on Geometry processing, Eurographics Association, Aire-la-Ville, Switzerland, Switzerland, 6170.

[12] I. Bilasco and J. Gensel and M. Villanova-Oliver and H. Martin, An MPEG-7 framework enhancing the reuse of $3 D$ models in Web3D '06: Proceedings of the eleventh international conference on 3D web technology, pp.65-74, 2006

[13] F. Pittarello and A. De Faveri Semantic description of $3 D$ environments: a proposal based on web standards. In Proceedings of the Eleventh international Conference on 3D Web Technology (Columbia, Maryland, April 18 - 21, 2006).

[14] S. Havemann and V. Settgast and R. Berndt and O. Eide and D. Fellner The Arrigo Showcase Reloaded - towards a sustainable link between $3 D$ and semantics in VAST: International Symposium on Virtual Reality, Archaeology and Intelligent Cultural Heritage proceedings, pp 125-132. 2008

[15] OpenSG OpenSG webpage http://opensg.vrsource.org/trac, last visited July 2009

[16] K. Rodriguez-Echavarria and D. Morris and D. Arnold Web Based Presentation of Semantically Tagged 3D Content for Public Sculptures and Monuments in the UK Web3D 2009: Proceedings of the 14th International Conference on 3D Web Technology 2009 